Egyptian Journal of Aquatic Biology \& Fisheries Zoology Department, Faculty of Science,

Ain Shams University, Cairo, Egypt.

ISSN $1110-6131$

Vol. 23(5): 121 - 133 (2019)

www.ejabf.journals.ekb.eg

\title{
Pentoxifylline and/or praziquantel reduce murine schistosomiasis mansoni histopathology via amelioration of liver functions
}

\author{
Amina Ibrahim ${ }^{1}$, Heba Abdel-Tawab ${ }^{2}$ and Taghreed Hussein ${ }^{3}$ \\ 1- Environmental Research and Medical Malacology Department, Theodor Bilharz \\ Research Institute; Giza; Egypt. \\ 2- Department of Zoology, Faculty of Science, Beni-Suef University, Beni-Suef, Egypt \\ 3- National Organization for Drug Control and Research, Cairo, Egypt \\ E-mail: aminamd.ibrahim@yahoo.com; aminamd_1982@yahoo.com
}

\section{ARTICLE INFO}

\section{Article History:}

Received: June 1, 2019

Accepted: Nov. 22, 2019

Online: Dec. 2019

\section{Keywords:}

Schistosoma mansoni

Pentoxifylline

Praziquantel

PTX treatment

Antifibrotic substance

Immunomodulatory

\section{ABSTRACT}

Murine schistosomiasis represented a good lab model of granulomatous hypersensitivity reaction which could be elicited by $S$. mansoni eggs trapped in the host tissues which might lead to liver fibrosis. Pentoxifylline (PTX) is an immunomodulatory and antifibrotic substance. The present investigation aimed to study the action of PTX (4 weeks post infection) either alone or combined with PZQ (7 wk PI) on fibrosis developed from murine hepatic schistosomal granulomatous reaction. The experiments were carried out on naïve C75BL/6 mice that were divided into three groups. Two control groups were used one without infection (normal) and the other was infected without treatment. Mice were sacrificed 12 weeks post infection.

PTX treatment alone caused a partial toxic effect on worm burden; egg count; and ameliorated the liver functions. But, it increased immature and dead eggs, while it decreased the mature ones. PZQ administration alone or in combination with PTX showed a highly significant reduction in worm burden, egg count, disappearance of immature egg stage and a high increase in dead eggs. Also, it caused a highly significant reduction in granuloma count, diameter and ameliorated liver functions.

In conclusion, according to the parasitological, histopathological and biochemical criteria of the present study, PTX can be successfully introduced into antischistosomal therapy as a potent antifibrotic agent with immunomodulatory properties in combination with PZQ.

\section{BACKGROUND}

Schistosomiasis is a poverty symptom that is prevalent in 78 countries with 92 $\%$ living in sub-Saharan Africa (World Health Organization, 2018). It is a widespread neglected tropical parasitic disease transmitted by snails (Ibrahim and Ghoname, 2018) caused by a digenian trematode of the genus Schistosoma and it is affected more than 261 million people worldwide (Feitosa et al., 2018; Ibrahim and Sayed, 2019). Schistosoma mansoni infection resulted in severe histopathological changes and functional damage in the liver of the host (Leo and Peter, 1998). These alterations include the deposition of schistosome eggs and pigments, increase in organ size and formation of granulomas surrounding the deposited eggs which may result in scaring, 
portal hypertension, haemorrhage and death (Gause et al., 2003). Liver fibrosis is a wound-healing process that occurs when the liver is injured chronically (Friedman, 2003). Hepatic stellate cells (HSC) are responsible for the excess production of extracellular matrix (ECM) components (Han, 2018). The activation of HSC; a key issue in the pathogenesis of hepatic fibrosis (Bartley et al., 2006); is mediated by various cytokines and reactive oxygen species released from the damaged hepatocytes and activated Kupffer cells (Iredale, 2003). Therefore, inhibition of HSC activation and its related subsequent events, such as increased production of ECM components and enhanced proliferation; are crucial goals for intervention in the hepatic fibrogenesis cascade (Bataller and Brenner, 2001).

Praziquantel is the cornerstone drug for the treatment of all species of Schistosoma (Vale et al., 2017), which has low toxicity; great efficacy and is easy to administer (Utzinger and Keiser, 2004). PZQ has rapid action against adult schistosome worms with changes in the tegument and muscular activity, possibly resulting from calcium ion flux within the worm (Vale et al., 2017). Although treatment with this drug was effective and inexpensive (Cioli and Pica-Mattoccia, 2003), frequent schistosome reinfection after treatment due to the relative resistance of the larval stages of S. mansoni to schistosomicide drugs (Silva et al., 2003), occurred in endemic areas. Also, Abouel-dahab and Elhussieny (2016) found that PZQ has little or no effect on the developing larvae of 3-21 days post infection. Beshay et al., (2019) stated that it was preferable to make combination of PZQ and antiinflammatory drugs in the treatment of murine schistosomiasis mansoni.

Pentoxifylline (PTX) is a synthetic derivative of methylxanthine (El-Lakkany et al., 2011) and had significant anti-fibrotic effects on experimentally induced schistosomal hepatic fibrosis (Khalifa and Nemenqani, 2014).

The main pharmacological action of PTX is to protect hepatocytes from excessive cytokines that are responsible for activation of HSC by inhibiting them. $\mathrm{Li}$ et al. (2016) confirmed that PTX prevents in vitro liver fibrosis induced by Schistosoma japonicum infection by inhibiting the Hedgehog $(\mathrm{HH})$ signaling pathway. Also, El-Lakkany and Nosseir (2007) stated that PTX has limited toxic effects on schistosome worms and eggs and thus, can be used as an adjuvant therapeutic tool with anti- helminthic drugs in treatment of human schistosomiasis (Abdel aziz et al., 2012). Rabia et al. (2010) and Mati et al. (2010) stated that the addition of antifibrotic drugs PTX or silymarin to PZQ potentiated an antipathological effect which minimized and ameliorated the liver fibrosis.

The aim of the present research is to investigate the effects of the administration of the antifibrotic agent PTX alone or associated with PZQ on the course of murine schistosomiasis mansoni, using different parasitological, histopathological and biochemical parameters.

\section{MATERIAL AND METHODS}

\section{Animals:}

Male C57BL/6 mice strain; 6-8 weeks (wk) old (18-20 g); were purchased from the Schistosome Biological Supply Program, Theodore Bilharz Research institute, Giza-Egypt (SBSP, TBRI) in accordance with international guidelines. Mice were maintained for $8 \mathrm{wk}$ in plastic cages in an animal room, at temperature ranging between $20-25^{\circ} \mathrm{C}$ and were fed Purina chaw (20\% protein) and given tap water. 


\section{Cercariae and infection of mice:}

Cercariae were obtained from the infected Biomphalaria alexandrina snails, from SBSP, TBRI. The number of cercariae was determined by using a dissecting microscope. Generally; three counts were made and the average was used to calculate the number of cercariae per $0.1 \mathrm{ml}$ of the cercarial suspension (Moore et al., 1977). Infection was done subcutaneously with 100 cercariae/mouse of the Egyptian strain of S. mansoni.

\section{Test drugs:}

Pentoxifylline (PTX) (Trental®, Aventis Pharma, Cairo, Egypt), was orally administered from day 30 post infection (PI) for 55 day (5 days/wk) at a dose of 360 $\mathrm{mg} / \mathrm{kg}$ body weight.

Praziquantel (PZQ) (Distocide ${ }^{\circledR}$, Epico Pharma, Cairo, Egypt) is a white powder insoluble in water. It was given orally, $7 \mathrm{wk}$ PI, at a dose of $500 \mathrm{mg} / \mathrm{kg} \mathrm{b} . \mathrm{wt}$ for 2 consecutive days, using a blunt stainless steel oral cannula. It was freshly prepared before use as $2 \%$ suspension in Cremophor-El (Sigma Chemicals Co. St. Louis Missouri).

\section{Experimental design:}

30 mice were divided into 3 groups each of 10 mice as follows:

1- Normal untreated mice.

2- Normal mice treated with PTX $(360 \mathrm{mg} / \mathrm{kg} /$ day $)$ for $8 \mathrm{wk}$.

3 - Normal mice treated with PZQ ( $500 \mathrm{mg} / \mathrm{kg} /$ day) for 2 consecutive days.

40 mice all infected with $100 \mathrm{~S}$. mansoni cercariae/ mouse, were divided into 4 groups, each of 10 mice as follows:

1- Infected untreated control (positive controls).

2- Infected-PTX treated mice. Mice received PTX at 4 wk PI for 8 wk (360 $\mathrm{mg} / \mathrm{kg} /$ day).

3- Infected-PZQ treated mice: Mice received PZQ (500mg/kg/day); 7 wk PI for 2 consecutive days.

4- Infected-PTX and PZQ treated mice. Mice received PTX at 4 wk PI for 8 wk, and were treated with PZQ; 7 wk PI for 2 consecutive days.

Mice of all experimental groups were sacrificed by cervical dislocation at 12 wk PI and were subjected to investigations.

5- Parasitological study:

5.1-Worm burden and distribution: Perfusion of hepatic and protomesentric vessels of mice was carried out according to (Duvall and DeWitt; 1967). Mice were sacrificed by cervical dislocation. No resort to general anesthesia or heparin was attempted, because of the hepatic shift they interfere with the interpretation of genuine drug effect.

5.2- Oogram pattern:

The percentage of eggs at different developmental stages in the small intestine was examined in three samples per animal and the mean of each stage per animal was obtained. Eggs were counted and classified into their stages of development according to (Pellegrino et al., 1962).

5.3- Egg count in liver and intestine tissues: The number of ova per gram hepatic or intestinal tissue was counted (Kamel et al., 1977).

6-Histopathological investigation:

Liver sample from each animal was preserved in $10 \%$ buffered formalin solution, till dehydrated; sectioned and stained with Ehrlich's hematoxylin and counter stained with eosin (Bancroft and Stevens, 1975). 


\section{Granulomas counting and Measurement of granuloma diameter:}

The number of granulomas in 5 successive fields (10x10) was counted and recorded. Measurement of granuloma diameter was done only for non-contiguous granulomas, each containing a single egg in the center using a calibrated ocular micrometer. The mean granuloma diameter (M.G.D) was calculated by measuring two diameters of the lesion at right angles to each other (Von Lichtenberg, 1962). The percent reduction in granuloma diameter/ treated group relative to the infected groups was calculated according to the following formula:

$$
\% \text { Reduction }=\frac{\text { M.G.D. of control group }- \text { M.G.D. of treated group }}{\text { M.G.D. of control group }} \times 100
$$

\section{Serum preparation and biochemical investigation:}

Mice were sacrificed by cervical dislocation and the blood was collected in plastic tubes not containing anticoagulant. Blood was allowed to stand at $37^{\circ} \mathrm{C}$ for 1 $\mathrm{hr}$, then over night at $4^{\circ} \mathrm{C}$, and centrifuged at $300 \mathrm{G}$ for 30 minutes (min). Sera were separated and heat-inactivated at $56^{\circ} \mathrm{C}$ for $30 \mathrm{~min}$ and stored in aliquots at $-20^{\circ} \mathrm{C}$, until use. Measurement of alanine aminotransferase (ALT) and aspartate aminotransferase (AST) were determined in serum according to the method described by (Reitman and Frankel, 1957).

\section{Statistical analysis:-}

Data are expressed as mean \pm standard error of mean $(\mathrm{X} \pm \mathrm{SEM})$. Comparison between two means was done using student's t-test (Snedecor and Cochran, 1991).

\section{RESULTS}

The present study revealed that treatment of S. mansoni infected mice with PTX alone caused an insignificant reduction in the mean total worm number, while, when the infected mice treated with PZQ alone or in combination with PTX after 4 wk of infection caused a highly significant reduction $(\mathrm{P}<0.001)$ (table 1$)$. Regarding the mean total number of ova in the intestinal vessels, there was a highly significant reduction in egg number $(\mathrm{P}<0.001)$ between the control group and the groups receiving $\mathrm{PTX}, \mathrm{PZQ}$ or $\mathrm{PTX}+\mathrm{PZQ}$. Also, in the hepatic vessels, a highly significant egg reduction $(\mathrm{P}<0.001)$ was observed in groups taking $\mathrm{PZQ}$ or $\mathrm{PTX}+\mathrm{PZQ}$, while, there was no significant decrease was recorded between the control group and the group receiving PTX (table 1).

Table 1: The effect of PTX and PZQ administrations each alone or in combinations with each other; on total number of worms and the number of ova/gm tissue in mice infected with $100 \mathrm{~S}$. mansoni

\begin{tabular}{|c|c|c|c|}
\hline \multirow[t]{2}{*}{ Animal groups } & \multirow{2}{*}{$\frac{\text { Mean total worm number }}{(\mathrm{X} \pm \mathrm{SEM})}$} & \multicolumn{2}{|c|}{ Mean total number of ova/gm tissue $(\mathrm{X} \pm \mathrm{SEM})$} \\
\hline & & Intestine & Liver \\
\hline a) Infected Control & $31.5 \pm 2.070$ & $64.7 \pm 2.582$ & $51.5 \pm 4.562$ \\
\hline b) Infected +PTX & $24.6 \pm 2.500$ & $48.6 \pm 4.728 * *$ & $45.7 \pm 5.394$ \\
\hline d) Infected + PZQ & $0.375 \pm 0.374 * *$ & $7.2 \pm 0.940 * *$ & $5.1 \pm 0.718 * *$ \\
\hline f) Infected +PTX+PZQ & $0.125 \pm 0.12 * *$ & $9.1 \pm 1.187 * *$ & $4.3 \pm 0.1829 * *$ \\
\hline *-Significant $(\mathrm{P}<0.01)$ & & & \\
\hline
\end{tabular}
cercariae; 4 wk PI and sacrificed 12 wk PI.

The present results showed that there was no significant difference in the percentage of immature eggs between the control group and PTX group. While, a very 
highly significant reduction $(\mathrm{P}<0.001)$ in this percentage between the control group and groups receiving PZQ or combination of PTX+PZQ was reported. Regarding the mature eggs, a highly significant decrease $(\mathrm{P}<0.001)$ was reported in all treated groups (PTX, PZQ or a combination of PTX + PZQ groups).

There was a significant increase $(\mathrm{P}<0.01)$ in the mean percentage of dead eggs between the control group and the group treated with PTX, PZQ or a combination of PTX+PZQ was reported (Table 2).

Table 2: The effect of PTX and PZQ administrations; each alone or in combinations with each other, on $\%$ egg developmental stages (Oogram) in mice infected with 100 S. mansoni cercariae; 4 wk PI and sacrificed $12 \mathrm{wk}$ PI.

\begin{tabular}{|c|c|c|c|}
\hline \multirow[t]{2}{*}{ Animal groups } & \multicolumn{3}{|c|}{$\%$ Egg developmental stages $(\mathrm{X} \pm \mathrm{SEM})$} \\
\hline & $\%$ Immature Ova & $\%$ Mature Ova & $\%$ Dead Ova \\
\hline a) Infected Control & $44.3 \pm 4.55$ & $47.7 \pm 4.640$ & $7.8 \pm 1.060$ \\
\hline b) Infected +PTX & $55.8 \pm 4.437$ & $32.2 \pm 2.144 * *$ & $11.8 \pm 1.381^{*}$ \\
\hline d) Infected + PZQ & $0.0 \pm 0.0 * * *$ & $2.2 \pm 1.292 * * *$ & $97.7 \pm 8.293 * * *$ \\
\hline f) Infected + PTX+ PZQ & $0.0 \pm 0.0 * * *$ & $1.6 \pm 1.224 * * *$ & $98.8 \pm 6.875^{* * *}$ \\
\hline
\end{tabular}

** Significant difference $(\mathrm{P}<0.001)$

$\mathrm{X}=$ mean total number. $\quad \mathrm{SEM}=$ standard mean error.

The present results indicated that there was a highly significant reduction $(\mathrm{P}<0.001)$ in the number of granuloma in the liver between groups that were taken PTX, PZQ and PTX+PZQ in comparison to the control group. Also, there was a very highly significant reduction $(\mathrm{P}<0.001)$ in the mean granuloma diameter in the liver between groups that were taken PTX, PZQ and PTX+PZQ in comparison to the control group (table 3).

Table 3: The effect of PTX, PZQ and combination of PTX and PZQ administrations on number of hepatic granuloma (NG) [in 5 successive power fields (10x10)] and mean diameter of granuloma (DG) in mice infected with $100 \mathrm{~S}$. mansoni cercariae; 4 wkPI and sacrificed 12 wkPI

\begin{tabular}{lllll}
\hline Animal groups & NG X \pm SEM & \% Reduction & Mean DG $(\mu \mathrm{m})$ X \pm SEM & \% Reduction \\
\hline a) Infected control & $13.4 \pm 0.927$ & --- & $342.047 \pm 7.208$ & --- \\
b) Infected +PTX & $9.2 \pm 0.374 * *$ & 31.3 & $278.1 \pm 3.700 * * *$ & 18.6 \\
d) Infected + PZQ & $8.2 \pm 0.374 * * *$ & 38.8 & $264.3 \pm 2.999 * * *$ & 22.7 \\
f) Infected +PTX+ PZQ & $8.0 \pm 0.447 * * *$ & 40.2 & $254.9 \pm 5.24 * * *$ & 25.4 \\
\hline
\end{tabular}

* Significant difference between group (a) and the next groups.

$\mathrm{X}=$ mean total number. $\quad \mathrm{SEM}=$ standard mean error.

Liver sections of infected untreated control showed circumscribed round granuloma formation which consisted of centrally located ovum $(\mathrm{O})$, surrounded by collagen fibers (C) and lymphoid cells (M), as well as, mononuclear leucocytes (arrow) (figure 1. B) as compared with the liver of normal mice (figure 1. A). In infected mice receiving PTX, around the granuloma there was Kupffer cells proliferation (arrow) in between the degenerated hepatocytes surrounding the central vein $(\mathrm{CV})$ (figure 1.C).

Infected mice subjected to PZQ showed hepatic granuloma formation with centrally located ova surrounded by fibrous tissue collagen and lymphocytes as well as mononuclear leucocytes, in an adhesive manner (figure 1. D). Kupffer cells proliferation with mononuclear leucocytes infiltration were observed in between the degenerated hepatocytes as well as in between the karyomegalic cells. In group receiving PTX+PZQ, mononuclear leucocytes inflammatory cells infiltration were observed in the portal area associated with dilatation in the central vein and collagen fibers were the most abundant component of the granuloma (figure 1.E and F). 

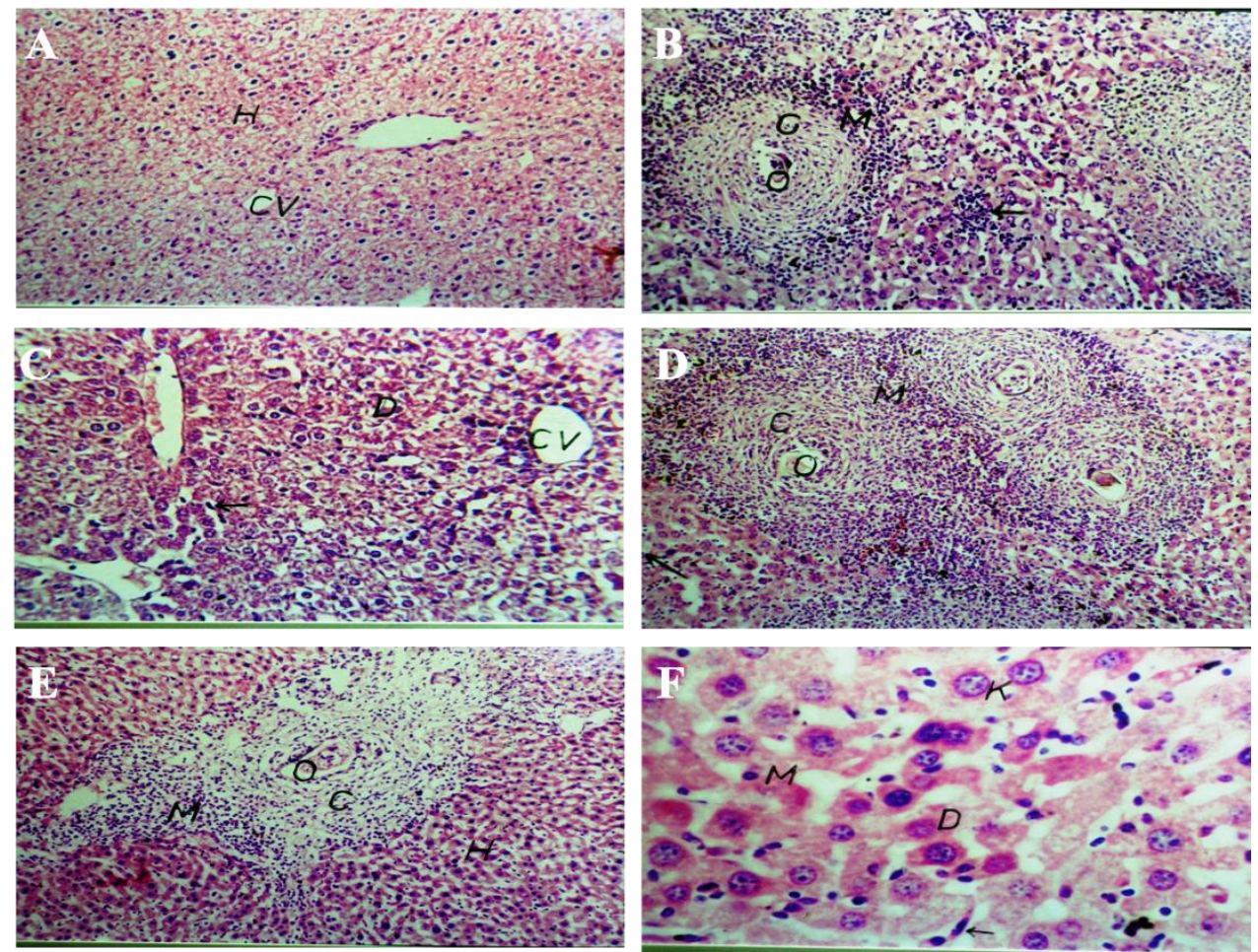

Fig. 1: Liver sections showing A) the normal histological structure of the central vein (CV) and surrounding hepatocytes $(\mathrm{H})(\mathrm{H} \& \mathrm{E}$ stain $\mathrm{x} 40)$. B) Showing circumscribed round granuloma formation of central ovum (O) surrounded by collagen (C) and macrophages as well as lymphocytes (M); while the other hepatic tissue showing focal as well as diffuse mononuclear leucocytes inflammatory cells infiltration (arrow) (H\&E stain $x$ 40). C) infected- PTX treated mice:-Showing Kupffer cells proliferation (arrow) in between the degenerated hepatocytes (D) surrounding the central vein (CV) (H\&E stain x 64). D) infected-PZQ treated group:- Showing granuloma formation with central ovum $(\mathrm{O})$ surrounded by collagen $(\mathrm{C})$ and more lymphoid cells $(\mathrm{M})$ and mononuclear leukocytes. Notice the multiple number of granuloma with peripheral zones adhesion and diffused kupffer cells (arrow) (H\&E stain x 40). E) Liver sections of infectedPTX+PZQ treated group:- Showing a massive number of mononuclear leukocytes inflammatory cells infiltration (M) in the portal area and normal hepatocytes (H) (arrow) (H\&E stain x 40) and F) Showing mononuclear leukocytes infiltration (M) with diffuse kupffer cells proliferation (arrow) in between the degenerated hepatocytes (D) and karyomegaly of some hepatocytes (K) (H\&E stain x 160).

The present investigation showed that a highly significant increase $(\mathrm{P}<0.001)$ between the normal groups and the infected untreated control group in the value of ALT and AST level was detected. Comparing between the infected control group and groups receiving PZQ or PTX $+\mathrm{PZQ}$ a very highly significant decrease $(\mathrm{P}<0.001)$ in the amount of ALT and AST was recorded. While, no significant decrease was observed in groups subjected to PTX (Figure 2).

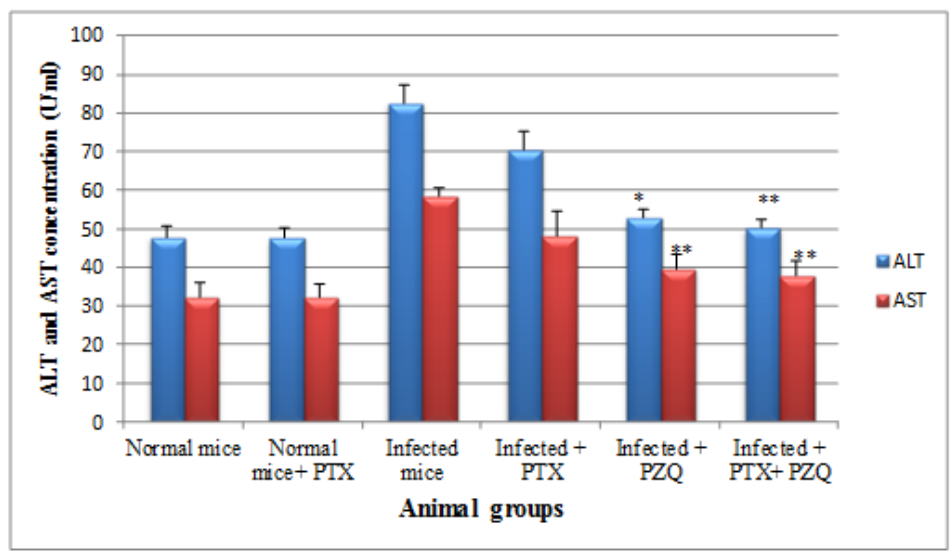

Fig. 2: The effect of PTX and PZQ administrations; each alone or in combinations with each other; on ALT and AST in mice infected with 100 S. mansoni cercariae; 4 wk PI and sacrificed 12 wk PI. 


\section{DISCUSSION}

The present study revealed that PTX treatment singly had a marked significant reduction in worm burden $(21.8 \%)$; ova/gm tissue in intestinal and hepatic vessels (24.8 in intestinal tissue and $11.5 \%$ in hepatic tissue; respectively). This coincides with the result of El-Lakkany and Nosseir (2007) who reported 22.6\% worm reduction. Also, the administration of PZQ alone or combined to PTX caused a high eradication percentage of worms, significant reduction in tissue egg load, in number and diameter of granulomas. The antischistosomal drug caused death of worms through making metabolic disorders, mechanical destruction and muscular contraction of the treated worms (García et al., 2006). Moreover, Martins-Leite et al. (2008) concluded that the chemotherapy with PZQ is effective in reducing the morbidity of the disease. Guirguis (2012) reported that reduction in the egg tissue count of infected host after PZQ treatment could be through hindering the process of oviposition. The percent reduction in the egg count was found to be higher in the intestinal tissue than in the hepatic tissue. This variation was attributed to excretion of some ova from the intestine prior to digestion and to hepatic shift of worms after treatment (Ebeid et al., 2008).

In the present work, PZQ either alone or combined with PTX caused the disappearance of immature egg stages; decrease in the number of mature eggs and increase in the number of dead eggs and this is in agreement with the findings of Botros et al. (1996). PTX alone caused an increase in immature and dead eggs and a decrease in the mature eggs. This was in accordance with the findings of El-Lakkany and Nosseir (2007) and Gouveia et al. (2019).

The manifestations of Schistosomiasis were attributable to granulomatous inflammation around parasite eggs (Abath et al., 2006) in host liver and intestine. The formation of granulomas depends predominantly on CD4+ T helper (Th) cells, specific for antigens secreted from viable schistosome larvae within eggs trapped in host tissue (Garcia et al., 2008); and represents a delayed-type hypersensitivity (Pearce, 2005). In the present study, the granuloma reached the maximal number $(13.4 \pm 0.927)$ and diameter $(342.04 \pm 7.208)$, at $8 \mathrm{wk}$ PI, in the infected untreated mice. The liver of this group showed collagen fibers and lymphoid cells as well as mononuclear leucocytes and there were diffuse proliferation of kupffer cells, pigmented cells and mononuclear leucocytes inflammatory cells infiltration in between the degenerated hepatocytes in association with karyomegally in the nuclei of the hepatocytes (Rojkind and Valadez, 1985; Ibrahim et al., 2014).

In the present work; a great improvement in the histopathological aspects in infected mice treated with PTX which caused a reduction in granuloma count and in granuloma diameter. The improvement of the histopathological situation of the liver was evidenced by the decreased number and size of granuloma and the disappearance of pathological changes in hepatocytes (Kamel et al., 2000). There was circumscribed round granuloma formation in the hepatic tissue with centrally located ova surrounded by abundant collagen, less cellular lymphoid cells and mononuclear and leucocytes diffuse kupffer cells proliferation in between the degenerated hepatocytes with normal histological collagen fibers. This agrees with the observation of El-Lakkany and Nosseir (2007). Khalifa and Nemenqani (2014) concluded that pentoxifylline (PTX) caused significant reductions in granuloma sizes, hepatic hydroxyproline and serum levels of leptin and transforming growth factor- $\beta 1$. PTX decreased the 
intragranulomatous eosinophil accumulation possibly due to its immunomodulatory capability; interfering in cellular recruitment and/or differentiation (Conceição et al., 2005).

The group treated with PZQ had a granuloma with centrally located ova surrounded by fibrous tissue collagen and lymphoid as well as mononuclear leucocytes were detected in the hepatic tissue in an adhesive manner. Kupffer cells proliferation with mononuclear leucocytes infiltration were observed in between the degenerated hepatocytes; as well as; in between the karyomegalic cells. This agrees with the finding of El-Banhawey et al. (2007) who concluded that PZQ was effective in reducing granuloma size in infected mice. The treatment of $S$. mansoni-infected mice with PZQ showed a minimal decrease in the number of granulomas two wk PI with diffused kupffer cells proliferation in between the degenerated hepatocytes (Kamel et al., 2000). Marked improvements of the histopathological changes were observed in group treated with PTX+PZQ, where, data recorded reduction in granuloma count and diameter. The histopathological change showed that the granuloma formation had mostly collagen content associated with dilatation in central vein and proliferated kupffer cells in between the hepatocytes (Farrag et al., 2015). El-Lakkany and Nosseir (2007) stated that treatments with PTX and/or PZQ ameliorated the liver functions. Also, Rabia et al. (2010) stated that the addition of antifibrotic drugs PTX or silymarin to PZQ ameliorated the liver status.

Serum enzymes (AST and ALT) are a helpful screening way for the detection of liver damage (Badrick and Turner, 2016). In the present work; the infected control mice showed a significant increase in serum ALT and AST than normal controls. Similarly, raised activities of these enzymes have been recorded by (Ezzat et al., 2011). This alteration seemed to be due to hepatic cell damage and impaired cell membrane permeability (Ahmed, 1995) or due to heavy schistosome egg deposition (Panic et al., 2017). The administration of PTX decreased the concentration of ALT and AST but insignificantly; compared to the infected untreated control group. Massart et al. (2012) stated that long-term PTX therapy achieves effectively sustained biochemical improvement. This correlates well with histological resolution of the disease. A tendency for the normalization of serum AST and ALT in this study; was noticed in group treated with PZQ, where, it caused a highly significant reduction in the concentration of both ALT and AST than infected group. This agrees with the findings of Utzinger et al. (2001). These decreased levels after PZQ administration might be due to loss of hepatic tissue proteins that follows hepatic necrosis (Badawy et al., 1996).

In this work, combined treatment of PTX with PZQ caused a highly significant decrease in liver enzymes as compared to the infected control group. This can be explained by the dual action of the antifibrotic action of PTX, in addition to its antioxidative properties, through inhibiting the free oxygen radicals of toxins released by eggs and worms or acting as a hydroxyl radical scavenger (Shukla and Gude, 2003) and the anti-helminthic drug. 


\section{CONCLUSION}

According to the parasitological, histopathological and biochemical criteria, the present findings indicate that PTX treatment for $8 \mathrm{wk}$ has a partial toxic effect on worms, eggs and it ameliorates the liver functions. The combination of PZQ and PTX can successfully be introduced into antischistosomal therapy as a potent antifibrotic agent with immunomodulatory properties in combination with PZQ. Other studies on the effect of PTX on substances that regulate fibrosis or participate in the generation of the liver extracellular matrix were necessary for the complementary evaluation and monitoring of the effect of PTX on the progression of schistosomal hepatic fibrosis.

\section{Declarations:}

Ethics approval and consent to participate:

Ethical approval had been granted approval by the Ethics Committee of Theodor Bilharz Research Institute (TBRI).

\section{Availability of data and materials:}

All the data obtained during the study are presented in this manuscript. Any further enquiries for additional information are available upon request from the corresponding author.

\section{Competing interests}

The authors declare that they have no competing interest.

\section{Authors' contributions}

AMI conceived and designed the study, performed the experiments, analyzed the data and wrote the first draft. AMI and TMH made the infection of the mice. AMI and HA revised and edited the manuscript. All authors read and approved the final manuscript.

\section{REFERENCES}

Abath, F.G.C.; Morais, C.N.L.; Montenegro, C.E.L.; Wynn, T.A. and Montenegro, S.M.L. (2006), Immunopathogenic mechanisms in schistosomiasis: What can be learnt from human studies?. Trends Parasitol, 22(2): 85-91.

Abdel-aziz, M.T.; Atta, H.M.; Roshdy, N.K.; Rashed, L.A.; Sabry, D.; Hassouna, A.A.; Aboul Fotouh, G.I.; Hasan, N.M.; Younis, R.H. and Chowdhury, J.R. (2012), Amelioration of Murine Schistosoma mansoni Induced Liver Fibrosis by Mesenchymal Stem Cells. J Stem Cells Regen Med. 2012; 8(1): 28-34.

Aboueldahab, M.M. and Elhussieny, E.A. (2016), Antiparasitic and physiological evaluation of Curcuma longa extract and/or PZQ on Schistosoma mansoni infected mice. International Journal of Advanced Research, 4 (6): 1020-1039.

Ahmed, S.A. (1995), A preliminary report on the prognostic value of selected diagnostic enzymes among certain malignant and schistosomal malignant patients. Journal of the Egyptian Society of Parasitology, 25: 659-76.

Badawy, A.; El-Badrawy, N.M.; Mansy, S.S.; Akl, M.M.; Abdel Hady, A.M.; Ebeid, F.A. and Hassan, M.M. (1996), Evaluation of Colchicine with or without Praziquantel therapy in the control of hepatic fibrosis in murine schistosomiasis. Pharmacological Research, 33: 319-325.

Badrick, T. and Turner, P. (2016), Review and Recommendations for the Component Tests in the Liver Function Test Profile. Indian Journal of Clinical Biochemistry, 31: 21-29.

Bancroft, J.D. and Stevens, A. (1975), Histopathological stains and their diagnostic uses. J Clin Pathol, 28(10): 841. 
Bartley, P.; Ramm, G.; Jones, M.; Ruddell, R.; Li, Y. and Mcmanus, D. (2006), A contributory role for activated hepatic stellate cells in the dynamics of Schistosoma japonicum egg-induced fibrosis. International Journal for Parasitology, 36: 993-1001.

Bataller, R. and Brenner, D.A. (2001), Hepatic Stellate Cells as a Target for the Treatment of Liver Fibrosis. Seminars in Liver Disease, 21: 437-452.

Beshay, E.V.N.; Rady, A.A.; Afifi, A.F. and Mohamed, A.H. (2019), Schistosomicidal; antifibrotic and antioxidant effects of Cucurbita pepo L. seed oil and praziquantel combined treatment for Schistosoma mansoni infection in a mouse model. Journal of Helminthology, 93 (3): 286-294.

Botros, S.S.; Doughty, B.L.; Shaker, Z.A.; Akl, M.M.; Sharmy, R.; Diab, T.M. and Hassanein, H.I. (1996), Efficacy of an antipathology vaccine in murine schistosomiasis administered with and without chemotherapy. International journal of immunopharmacology, 18: 707-18.

Cioli, D. and Pica-Mattoccia, L. (2003), Praziquantel. Parasitology research, 90 (1): S3-9.

Conceição, M.J.; Pino, A.; Reis, L.F.; Lenzi, H.L.; Pelajo, M.; Takiya, C.M.; Ventura, T.G.; Souza, S.O. and Pereira, M.J.S. (2005), Quantitave and qualitative interferences of pentoxifillyne on hepatic Schistosoma mansoni granulomas: effects on extracellular matrix and eosinophil population. Memórias do Instituto Oswaldo Cruz, 96: 107-112.

Duvall, R.H. and DeWitt, W.B. (1967), An improved perfusion technique for recovering adult schistosomes from laboratory animals. The American journal of tropical medicine and hygiene, 16: 483-6.

Ebeid, F.A.; El-Lakkany, N.M.; Seif, S.H.; Botros, S.S. and Hammam, O.A. (2008), Schistosoma haematobium (Egyptian Strain): Rate of Development and Effect of Praziquantel Treatment. Journal of Parasitology, 94: 386-394.

El-Banhawey, M.A.; Ashry, M.A.; El-Ansary, A.K. and Aly, S.A. (2007), Effect of Curcuma longa or parziquantel on Schistosoma mansoni infected mice liver Histological and histochemical study. Indian Journal of Experimental Biology, 45: 877-889.

El-Lakkany, N.; and Nosseir, M. (2007), Pharmacodynamics of pentoxifylline and/or praziquantel in murine schistosomiasis mansoni. Apmis, 115: 184-194.

El-Lakkany, N.; Seif el-Din, S. and Ebeid, F. (2011), The use of pentoxifylline as adjuvant therapy with praziquantel downregulates profibrogenic cytokines, collagen deposition and oxidative stress in experimental schistosomiasis mansoni. Experimental Parasitology, 129: 152-157.

Ezzat, A.; Seif el-Din, S. and Ebeid, F. (2011), Effect of Praziquantel Treatment on the Activities of Some Liver Microsomal Enzymes in Mice Infected with Schistosoma mansoni. Arzneimittelforschung, 51: 325-331.

Farrag, E.M.; Mohamed, A.M.; Kadry, S.M.; Mahmoud, A.H.; Farrag, A.H. and Fayed, D.B. (2015), Impact of Citharexylum Quadrangular Chloroform extract and micronutrient on Praziquantel in Schistosoma mansoni infected mice. American Journal of Life Sciences, 3 (2): 62-70.

Feitosa, K.A.; Zaia, M.G.; Rodrigues, V.; Castro, C.A.; Correia, R.O.; Pinto, F.G.; Rossi, K.N.; Avó, L.R.; Afonso, A. and Anibal, F.F. (2018), Menthol and menthone associated with acetylsalicylic acid and their relation to the hepatic fibrosis in Schistosoma mansoni infected mice. Frontiers in Pharmacology, 8: 19.

Friedman, S.L. (2003), Liver fibrosis- from bench to bedside. Journal of hepatology, 
38(1): S38-53.

García, N.; Isturiz, G.; Aular, S. and Incani, R.N. (2006), The efficacy of human schistosomicide treatment may depend on the rate of transmission. Parasitology Research, 98: 545-549.

Garcia, T.C.M.; Fonseca, C.T.; Pacifico, L.G.; Durães, F.V.; Marinho, F.A.; Penido, M.L.O.; Caliari, M. V.; Melo, A.L.; Pinto, H.A.; Barsante, M.M.; Cunha-Neto, E. and Oliveira, S.C. (2008), Peptides containing T cell epitopes, derived from Sm14, but not from paramyosin, induce a Th1 type of immune response, reduction in liver pathology and partial protection against Schistosoma mansoni infection in mice. Acta Tropica, 106: 162-167.

Gause, W.; Urban, J.F. and Stadecker, M.J. (2003), The immune response to parasitic helminths: insights from murine models. Trends in immunology, 24: 269-77.

Gouveia, M.J.; Brindley, P.J.; Rinaldi, G.; Gärtner, F.; Costa, J.M. and Vale, N. (2019), Combination Anthelmintic/Antioxidant activity against Schistosoma mansoni. Biomolecules, 9 (2): 54.

Guirguis, F. (2012), Efficacy of Praziquantel and Ro 15-5458, a 9-Acridanonehydrazone Derivative, against Schistosoma haematobium. Arzneimittelforschung, 53: 57-61.

Han, C.Y. (2018), Update on FXR biology: Promising therapeutic target?, Multidisciplinary Digital Publishing Institute (MDPI). Int. J. Mol. Sci. , 19(7): 2069.

Ibrahim, A.M. and Ghoname, S.I. (2018), Molluscicidal impacts of Anagallis arvensis aqueous extract on biological, hormonal, histological and molecular aspects of Biomphalaria alexandrina snails. Experimental Parasitology, 192: 36-41.

Ibrahim, A.M. and Sayed, D.A. (2019), Toxicological impact of oxyfluorfen $24 \%$ herbicide on the reproductive system, antioxidant enzymes and endocrine disruption of Biomphalaria alexandrina (Ehrenberg, 1831) snails. Environ Sci Pollut Res, 26: 7960-7968.

Ibrahim, J.; Mohammed, A.; Hussien, N.; El-Shennawy, A.; Noshy, M. and Abbas, M. (2014), In vivo antioxidant and antigenotoxic evaluation of an enaminone derivative BDHQ combined with praziquantel in uninfected and Schistosoma mansoni infected mice. Journal of Applied Pharmaceutical Science, 4 (5): 25.

Iredale, J.P. (2003), Cirrhosis: new research provides a basis for rational and targeted treatments; BMJ; 327; 143-147.

Kamel, G.; Metwally, A.; Guirguis, F.; Nessim, N. and Noseir, M. (2000), Effect of a Combination of the New Antischistosomal Drug Ro 15-5458 and Praziquantel on Different Strains of Schistosoma mansoni Infected Mice. Arzneimittelforschung, 50: 391-394.

Kamel, I.A.; Cheever, A.W.; Elwi, A.M.; Mosimann, J.E. and Danner, R. (1977), Schistosoma mansoni and S. haematobium infections in Egypt. I. Evaluation of techniques for recovery of worms and eggs at necropsy. The American journal of tropical medicine and hygiene, 26: 696-701.

Khalifa, E.A. and Nemenqani, D.M. (2014), Efficacy of pentoxifylline as an antifibrotic drug in experimental murine schistosomal hepatic fibrosis. Journal of the Egyptian Society of Parasitology, 44: 475-88.

Leo, X.L. and Peter, F.W. (1998), Helminth infections: In: "Harrison's Principles of Internal Medicine".14th ed.; McGraw-Hill. Co. Inc. USA. 1: 1206 - 1227.

Li, H.; Hua, J.; Guo, C. X.; Wang, W. X.; Wang, B. J; Yang, D. L.; Wei, P. and Lu, Y. P. (2016), Pentoxifylline inhibits liver fibrosis via hedgehog signaling pathway. Journal of Huazhong University of Science and Technology, 36: 372- 
376.

Martins-Leite, P.; Gazzinelli, G.; Oliveira, L.F.; Gazzinelli, A.; Malaquias, L.C.C.; Oliveira, R.; Teixeira-Carvalho; A. and Silveira, A.M.S. (2008), Effect of Chemotherapy with Praziquantel on the Production of Cytokines and Morbidity Associated with Schistosomiasis Mansoni. Antimicrobial Agents and Chemotherapy, 52: 2780-2786.

Massart, J.; Robin, M.; Noury, F.; Fautrel, A.; Lettéron, P.; Bado, A.; Eliat, P. and Fromenty, B. (2012), Pentoxifylline aggravates fatty liver in obese and diabetic ob/ob mice by increasing intestinal glucose absorption and activating hepatic lipogenesis; British Journal of Pharmacology. 165: 1361-1374.

Mati, V.L.T.; Freitas, R.M.C.; Melo, A.L. (2010), Effects of pentoxifylline during Schistosoma mansoni infection in Swiss mice: an analysisof worm burden, fecundity and liver histopathology. Journal of Helminthology, 84: 348-354.

Moore, D.L.; Grove, D.I. and Warren, K.S. (1977), The Schistosoma mansoni egg granuloma: Quantitation of cell populations. The Journal of Pathology, 121: 4150.

Panic, G.; Ruf, M.T. and Keiser, J. (2017), Immunohistochemical Investigations of Treatment with Ro 13-3978; Praziquantel; Oxamniquine; and Mefloquine in Schistosoma mansoni-Infected Mice. Antimicrobial Agents and Chemotherapy, 61(12). doi: 10.1128/AAC.01142-17.

Pearce; E.J. (2005); Priming of the immune response by schistosome eggs.

Pellegrino, J.; Oliveira, C.A.; Cunha, A.S. and Faria; J. (1962), New Approach to the Screening of Drugs in Experimental Schistosomiasis Mansoni in Mice. The American Journal of Tropical Medicine and Hygiene, 11: 201-215.

Rabia, I.; Nagy, F.; Ali, E.; Mohamed, A.; El-Assal, F. and El-Amir, A. (2010), Effect of treatment with antifibrotic drugs in combination with PZQ in immunized Schistosoma mansoni infected murine model. International Journal of Infectious Diseases, 14: S16-S17.

Reitman, S. and Frankel, S. (1957), A colorimetric method for the determination of serum glutamic oxalacetic and glutamic pyruvic transaminases. American journal of clinical pathology, 28: 56-63.

Rojkind, M. and Valadez, G. (1985), Regulation of fibroblast proliferation by Kupffer cells and monocytes. Ciba Foundation symposium, 114: 208-21.

Shukla, V. and Gude, R.P. (2003), Amelioration of B16F10 melanoma cells induced oxidative stress in DBA/2 mice by pentoxifylline. Journal of experimental \& clinical cancer research, 22: 407-10.

Silva, L.M.; Menezes, R.M.C.; Oliveira, S.A. and Andrade, Z.A. (2003), Chemotherapeutic effects on larval stages of Schistosoma mansoni during infection and re-infection of mice. Revista da Sociedade Brasileira de Medicina Tropical, 36: 335-341.

Snedecor, W. and Cochran, G. (1991), Statistical methods; 8th Edition. WileyBlackwell, 503 Pages; https://trove.nla.gov.au/work/10694367 (Access Date: 2019-3-2).

Utzinger, J.; Chollet, J.; You, J.; Mei, J.; Tanner, M. and Xiao, S. (2001), Effect of combined treatment with praziquantel and artemether on Schistosoma japonicum and Schistosoma mansoni in experimentally infected animals. Acta tropica, 80: 9-18.

Utzinger, J. and Keiser, J. (2004), Schistosomiasis and soil-transmitted helminthiasis: common drugs for treatment and control. Expert Opinion on Pharmacotherapy, 5: 263-285. 
Vale, N.; Gouveia, M.; Rinaldi, G.; Brindley, P.J. and Correia, J.M. (2017), Praziquantel for schistosomiasis, single drug revisited metabolism. Antimicrobial Agents and Chemotherapy, 61: 1-16.

Von Lichtenberg (1962), Host response to eggs of S. mansoni. I. Granuloma formation in the unsensitized laboratory mouse. The American journal of pathology, 41: 711-31.

World Health Organization (2018), Schistosomiasis - Epidemiological Situation; https://www.who.int/news-room/fact-sheets/detail/schistosomiasis (Access Date: 2019-2-15). 